http://jmscr.igmpublication.org/home/ ISSN (e)-2347-176x ISSN (p) 2455-0450 crossref DOI: https://dx.doi.org/10.18535/jmscr/v8i8.23

\title{
Quality of sleep and sleep disturbance after mild and moderate traumatic head injury
}

\author{
Authors \\ Mugunthan Karalmarks ${ }^{1}$, Dr Sathish ${ }^{2}$, Dr Thanga Thirupathi Rajan ${ }^{3}$, Dr Nadish ${ }^{4}$ \\ ${ }^{1}$ MBBS 3rd year undergraduate, Saveetha Medical College, Chennai, Tamil Nadu, India \\ ${ }^{2}$ Professor of Neurosurgery Department, Saveetha Medical College, Chennai, Tamil Nadu, India \\ ${ }^{3}$ HOD of Neurosurgery Department, Saveetha Medical College, Chennai, Tamil Nadu, India \\ ${ }^{4}$ Professor of Neurosurgery Department, Saveetha Medical College, Chennai, Tamil Nadu, India
}

\begin{abstract}
Objective: To determine the incidence and severity of sleep disturbance in mild and moderate head injury patients.

Method: A cross sectional observational study done using the Pittsburgh sleep quality index (PSQI) questionnaire to assess sleep quality of head injury patients. The sleep quality of each individual is evaluated and scored according to the PSQI scale. Sleep quality of mild and moderate head injury patients were analysed and compared.

Result: This study demonstrates that the moderate head injury patients have decreased quality of sleep compared to mild head injury patients.

Conclusion: Sleep disruption are a common commodity after the traumatic head injury, as this has been supported by the study done and this also states that the influence of moderate head injury plays a more vital role compared to the mild traumatic head injury. Hence, there forth concluding that sleep disturbance are more commonly associated with moderate head injury.
\end{abstract}

\section{Introduction}

Traumatic brain injury is a non degenerative, non congenital insult to the brain from an external mechanical force, causing diminished or altered state of consciousness and leading to permanent or temporary impairment of cognitive, physical, and psychological. The assessment of patients with head injury is done by the Glasgow coma scale and is classified into mild, moderate and severe. The classification of the mild, moderate head injury is usually based on the patient's neurological status and imaging (table 1$)^{[1,2]}$.

Classification of head injury: (Table 1)

Table 1 Classification of traumatic brain injury

\begin{tabular}{|l|c|c|}
\hline Mild head injury & Moderate head injury & Severe head injury \\
\hline Normal imaging & Normal or abnormal imaging & Abnormal imag ing \\
\hline loss of consciousness 0-30 min. & LOC $>30 m i n<24 \mathrm{~h}$ & LOC $>24 \mathrm{~h}$ \\
\hline Alteration of consciousness (AOC): up to 24h & AOC $>24 \mathrm{~h}$ & AOC $>24-48 \mathrm{~h}$ \\
\hline Post traumatic amnesia (PTA): 0 - 1 day & PTA $>1$ and $<7$ days & PTA $>$ days \\
\hline
\end{tabular}


Sleep disturbances and the decreased quality of sleep are the most prevalent and persistent squeal of mild and moderate head injury. The sleep disturbances can develop acutely following injuries or emerge later during recovery, at times persisting after years ${ }^{[3]}$.

The etiology for the sleep disturbance and decreased sleep quality that is associated with mild and moderate head injury is due to any of the following reasons that include direct injury to the brain tissue by the acceleration or deceleration forces or the cellular events that are caused by hypoxia or raised intracranial pressure ${ }^{[4]}$.

Decreased quality of sleep and sleep disturbance due to mild and moderate head injury have shown to aggravate the psychiatric disorders affecting the mood and behaviour of certain patients.

The treatment for mild and moderate head injury is usual an conservative mode of treatment,

Our study is aimed at evaluating the sleep disturbance and quality of sleep in mild and moderate head injury patients using the Pittsburgh sleep quality index (PSQI).

\section{Methodology}

A cross sectional observational study done using the structured questionnaire called Pittsburgh Sleep quality index (PSQI) questionnaire on the100 patients of both sexes and all ages who have had a mild and moderate head injury and were on conservative treatment in Saveetha medical college and hospital. The Data were collected from the medical records of the hospital from June 2018 to June 2019 at the department of neurosurgery, Saveetha medical college and hospital. The questions are based on the sleep quality and any other sleep disturbances after the injury. And each question is graded and evaluated and a final score is obtained using the grading system given by the PSQI and the assessment of the sleep quality of each individual is done. And further the comparison of sleep quality and disturbance in mild head injury and moderate head injury is done. The case study included 100 patients enrolled, out of which the patients were grouped into those with mild head injury and moderate head injury based on classification (table 1). The Mild and moderate head injury patients with different mode of injury such as RTA, falling from a height, and being hit by object, assault. The PSQI questionnaire was asked to the patients by interactive voice response via telephone and the scores are entered. The global PSQI score is calculated by totalling the seven component scores, providing an overall score ranging from 0 to 21 , a total score of 5 or greater indicates poor sleep quality, and a score lower than 5 is regarded as normal ${ }^{[5]}$

\section{Result}

Out Of the 100 cases in our study, 50 of them had moderate head injury and 50 of them with a mild head injury all patients were conscious oriented with GCS-15/15 and no focal neurological deficits. Patients with sleeping disorders or psychiatric problems prior to the injury were excluded.

The data collection done in our institution revealed the following: (Chart 1)

Total number of cases collected : 100

Cases of Mild head injury : 50

Cases of Moderate head injury : 50

\section{NO.OF PATIENTS}

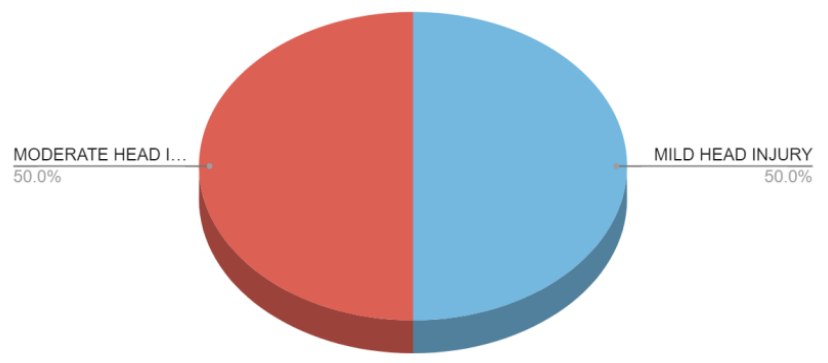

Pie Chart 1: Patients in Mild and Moderate Head Injury

The study conducted demonstrated that 66 out of the 100 patients with mild and moderate head injury had an PSQI value greater than 5, which indicates that they are suffering from some kind of 
sleep disturbance and have a poor quality of sleep after the injury. Evaluation of the PSQI also revealed that among the 50 mild head injury patient 24 of them had a value more than 5 (Chart 3 ), and therefore demonstrated poor quality of sleep. It also revealed that among the 50 moderate head injury patient $42(84 \%)$ of them had PSQI value more than 7 (Chart 2 ) which stated that the patients have a poor quality of sleep. hence our study revealed that the moderate head injurypatients had significantly more sleep disturbance than the mild head injury patients. This study has been analysed using the chi- square and the pi- value [p-0.000145] which shows the significance of sleep disturbance in moderate head injury. Even patients with mild head injury had considerable sleep disturbance with $48 \%$ of them have PSQI score more than 5 though they have no sleep trouble due to any pain or waking up in the middle of the night (Chart 4).

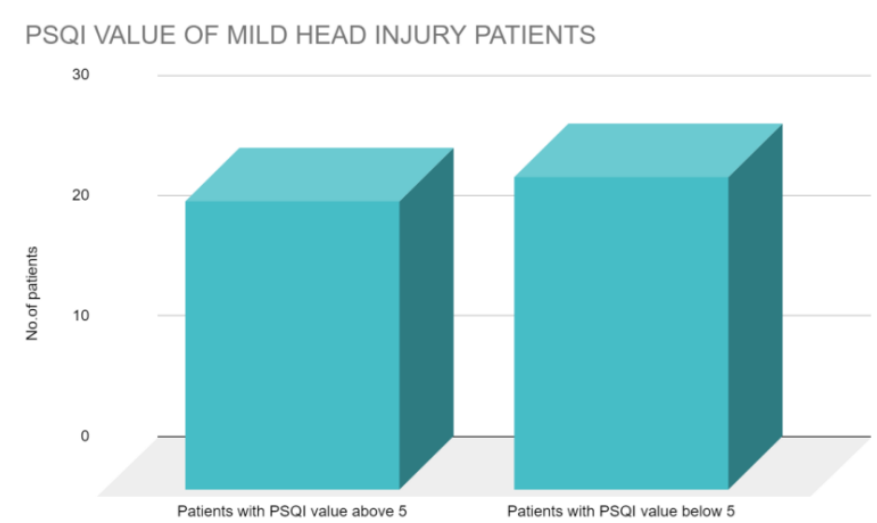

Flow Chart 2: PSQI Value in Mild Head Injury Patients

PSQI VALUE OF MODERATE HEAD INJURY PATIENTS

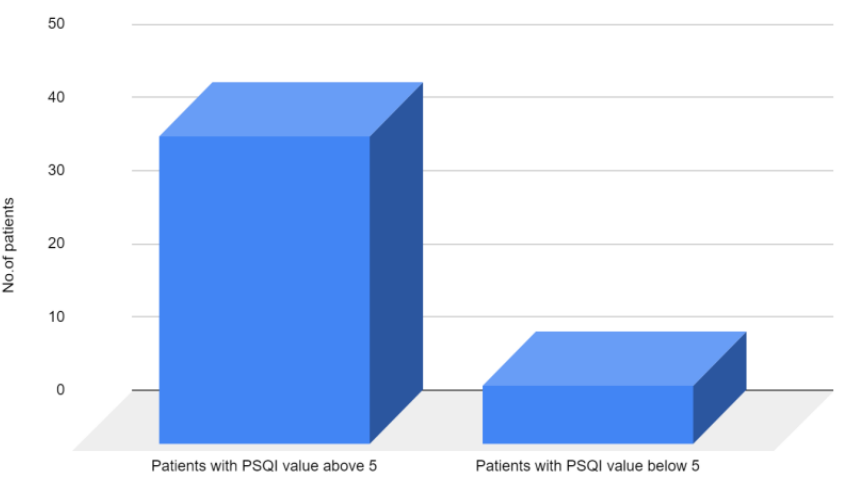

Flow Chart 3: PSQI Value in Moderate Head Injury Patients

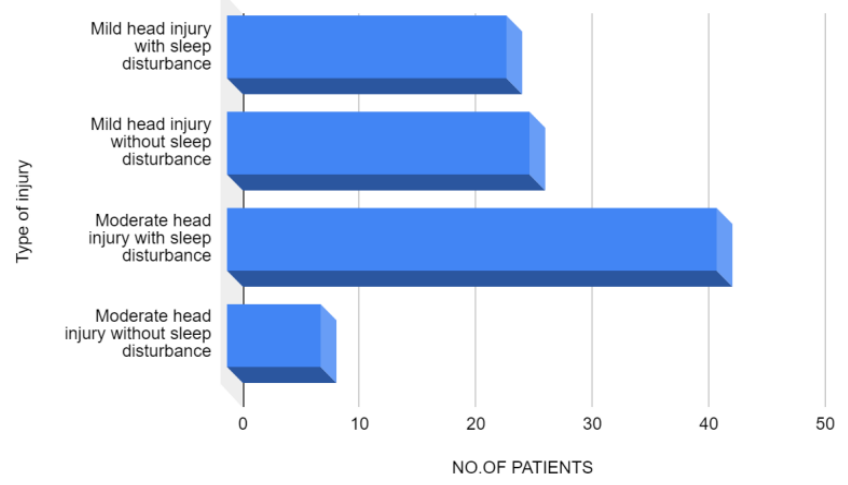

Flow Chart 4: Comparison of Sleep Quality in Mild and Moderate Head Injury Patients

\section{Discussion}

In our cross sectional study a total of 66 patients among the 100 enlisted patients experience sleep disturbance and decreased quality of sleep after the injury, Of which the majority is contributed by the moderate head injury patients. According to the study by Bauman et al in 2007, showed that 72 $\%$ of the moderately injured patients experience more sleep disturbance than the $42 \%$ of the mild injured patients ${ }^{[6]}$. Sleep disturbance were more common in the moderate head injury group than the mild head injury patients, with significantly $\mathrm{p}$ value $(0.000145)$. The subjects with moderate head injury/trauma have found to show the symptoms of insomnia and increased common sleep disorders ${ }^{[7]}$. Impairment of neuronal plasticity, metabolic alteration, loss of vascular homeostasis has been found to play a major role in the aetiology of sleep disruption in the traumatic patients ${ }^{[8]}$. Poor sleep maintenance was one of the major common sleep disturbances that has been noticed in the patients with moderate head injury $^{[9]}$. This study which was done by wick wire et al 2016 suggested that there are certain cases of mild injury that disrupt the sleep pattern and quality of sleep ${ }^{[10]}$. Similarly in our study, mild head injury had considerable sleep disturbance with $48 \%$ of them having PSQI more than 5. Chan and Feinstein classified the sleep disturbance into acute and chronic phase, the acute phase is felt on anywhere from one week to six months post injury and the chronic phase is anywhere from six 
months to one year, further they reported that the more severe type of injuries (moderate / severe injury) had been prevalent in causing both or chronic phase of sleep disruption than the mild cases. Drummond SP, Avalon 1, Orff HJ suggested from their study that there are multiple factors that contribute to the sleep disturbance caused by the head injury which brings about a delay in the recovery and resolution of the patient's illness ${ }^{[11]}$. Sleep disturbance among soldiers with combat related trauma, this study done by Jacob Colleen, Nicholas Orr. Suggested that the soldiers with mild and moderate head injury were found to have sleep disturbance and complaints. Thus this report suggests the role of mild and moderate head injury in the quality of sleep in an individual.

As the results obtained from our study relates to the results of previous studies and also share some similarities between them, which states that there are only few or rare cases of mild head injury that has been reported to cause sleep disturbance and the majority of the value from the PSQI also suggest that the moderate head injury has been reportedly associated with the sleep disturbance. The PSQI value of 42 patients out 50 has been found to be more than 5 , which states that there is some kind of sleep disturbance that a patient has been suffering, and of mild injury only 24 out of 50 has been reported to have a score higher than 5. Therefore, this has been accredited with the report that both moderate and mild head injury patients are associated with the sleep disturbance and decreased quality of sleep. While moderate head injury patients have significantly decreased quality of sleep and sleep related disorders compared to mild head injury patients.

\section{Conclusion}

Sleep disturbance is common entity seen in head injury patients. From our study we found that is a strong association between the traumatic head injury and sleep disturbance and decreased quality of sleep. It has been determined from the above study that moderate head injuries have a more association and influence on the sleep quality of the individual after the injury than the mild head injury patients. Hence the quality of sleep and sleep related disorders seem to be directly proportional to the severity of head injury. Thus, we conclude the study by stating that the moderate head injury patients suffer more sleep disturbance and decreased quality sleep.

\section{Reference}

1. Baumann C., Worth E., Stocker R., Ludwig S., Bassett C. Sleep-wake disturbances 6 months after traumatic brain injury: A prospective study. Brain. 2007; 130:1873-1883. doi: 10.1093/brain/awm109.

2. Maas A., Stocchetti N., Bullock R. Moderate and severe traumatic brain injury in adults. Lancet Neurol. 2008;7:728-741. doi: 10.1016/S1474-4422(08)70164-9.

3. Zeitzer J., Friedman L., OHara R. Insomnia in the context of traumatic brain injury. J. Rehabil. Res. Dev. 2009;46:827. doi: 10.1682/JRRD.2008.08.0099.

4. Andriessen T., Jacobs B., Vos P. Clinical characteristics and pathophysiological mechanisms of focal and diffuse traumatic brain injury.

5. Viola-Saltzman M., Watson N. Traumatic Brain Injury and Sleep Disorders.

6. Castriotta R., Murthy J. Sleep Disorders in Patients with Traumatic Brain Injury.

7. Wiseman-Hakes C., Murray B.J., Mollayeva T., Gargaro J., Colantonio A. A Profile of Sleep Architecture and Sleep Disorders in Adults with Chronic Traumatic Brain Injury. J. Sleep Disord. Ther. 2015;5:224. doi: 10.4172/21670277.1000224.

8. Ponsford J., Parcel D., Sinclair K., Roper M., Rajaratnam S. Changes in Sleep Patterns Following Traumatic Brain Injury. Neurorehabil. Neural. Repair. 2013;27:613-621 
9. Shekleton J., Parcel D., Redman J., Phipps-Nelson J., Ponsford J., Rajaratnam S. Sleep disturbance and melatonin levels following traumatic brain injury. Neurology. 2010;74:1732-1738. doi: 10.1212/WNL.0b013e3181e0438b.

10. Grima N., Ponsford J., Rajaratnam S., Mansfield D., Pase M. Sleep Disturbances in Traumatic Brain Injury: A MetaAnalysis. J. Clin. Sleep Med. 2016;12:419-428. doi: 10.5664/jcsm.5598.

11. Imbach L.L., Valko P.O., Li T., Maric A., Symeonidou E.R., Stover J.F., Bassetti C.L., Mica L., Werth E., Baumann C.R. Increased sleep need and daytime sleepiness 6 months after traumatic brain injury: A prospective controlled clinical trial. Brain. 2015;138:726-735. doi: 10.1093/brain/awu391. 